\title{
Anti-Fat Prejudice Reduction: A Review of Published Studies
}

\author{
Sigrún Daníelsdóttir ${ }^{\mathrm{a}}$ Kerry S. O’Brien ${ }^{\mathrm{b}}$ Anna Ciao $^{c}$ \\ a Division of Psychiatry, Landspítali-University Hospital, Reykjavík, Iceland \\ ${ }^{\text {b }}$ School of Psychological Sciences, University of Manchester, UK \\ ${ }^{c}$ Department of Psychology, University of Hawaii at Manoa, Honolulu, HI, USA
}

\section{Key Words}

Obesity - Anti-fat - Prejudice - Reduction - Weight bias . Stereotype · Stigma

\section{Summary}

Prejudice against those who are perceived as 'fat' or obese (anti-fat prejudice) is rife, increasing, and associated with negative outcomes for those targeted for such treatment. The present review sought to identify and describe published research on interventions to reduce anti-fat prejudice. A systematic search of relevant databases (e.g. PsychInfo, PubMed, Scopus) found 16 published studies that had sought to reduce anti-fat prejudice. Most notable was the lack of research on interventions for reducing anti-fat prejudice. Methodological problems that limit the interpretability of results were identified in the majority of studies found. Interventions employing more rigorous experimental designs provided at best mixed evidence for effectiveness. Although several studies reported changes in beliefs and knowledge about the causes of obesity, reductions in anti-fat prejudice did not typically accompany these changes. Anti-fat prejudice interventions adopting social norm- and social consensusbased approaches appear encouraging but are scarce. The lack of prejudice reduction following most interventions suggests that psychological mechanisms other than, or additional to, those being manipulated may underpin anti-fat prejudice. New directions for researching anti-fat prejudice are suggested. Given the strength of antipathy displayed toward those who are perceived as 'fat' or obese, research in this area is urgently required.

\section{Introduction}

Rates of overweight and obesity are increasing worldwide, with a majority of the adult population in westernized nations classifieds as either overweight or obese [1]. Juxtaposed with the increasing weight of the population is a somewhat counter-intuitive increase in prejudice towards people perceived as being 'fat' [2-5]. Anti-fat prejudice has been reported in numerous general life settings [6-23] and appears so ingrained and normative that parents have been shown to discriminate, whether consciously or unconsciously, against their own overweight daughters, specifically when it came to providing financial support for pursuing college education [7]. Indeed, while most people may deny knowingly holding anti-fat prejudices, newer measures of anti-fat prejudice that bypass socially desirable responding and/or tap into implicit attitudes show that people automatically associate negative attributes and beliefs with obese people [20]. Weight-based discrimination, the behavioral manifestation of anti-fat prejudice, has increased by $66 \%$ over the past decade with prevalence rates now comparable to race-based prejudice $[2,5]$. Additionally, research shows that the ill feeling displayed toward those perceived as overweight or obese exceeds that displayed towards other common target groups for mistreatment (i.e. Muslims and homosexuals [4]). Recent analyses of both the print and screen media $[15,16]$ reveal that obese people are also portrayed more negatively in the media than non-obese people. Specifically, obese individuals are consistently depicted as unattractive and lacking self control.

Anti-fat prejudice, also referred to in the literature as weight stigma, weight bias and anti-fat attitudes, is a negative attitude toward (dislike of), belief about (stereotype), or behavior against (discrimination) people perceived as being

\begin{tabular}{ll}
\hline KARGER & $\oplus$ 2010 S. Karger GmbH, Freiburg \\
Fax +497614520714 & Accessible online at: \\
Information@Karger.de & www.karger.com/ofa \\
www.karger.com &
\end{tabular}


'fat'. We use the term anti-fat prejudice over other terms as it maintains consistency with terminology used in the social psychology literature on hostility towards others (e.g. race, Muslims, gays) and also encompasses diverse constructs such as beliefs, attitudes, stereotypes and behaviors. Additionally, the most widely used measures of explicit anti-fat prejudice have the word 'anti-fat' in their title [8,9]. The term anti-fat prejudice also seems to capture the emotive negativity expressed towards those perceived as overweight, obese or 'fat', and leaves the public in no doubt about its important position alongside other prominent forms of prejudice such as racism and sexism. While we use the term anti-fat prejudice throughout this article, we adopt the terminology used by the respective authors when describing their work.

The study of prejudice and discrimination (e.g. directed toward race, religion, gender and sexuality) has dominated social psychology in terms of sheer research volume; however, comparatively the study of anti-fat prejudice is in its infancy. As with other emerging fields the literature in this area has largely been descriptive [17] and conducted predominantly in education, health, and employment settings where anti-fat prejudice appears pronounced. Research examining health professionals (e.g. doctors, dietitians, exercise scientists, nurses and psychologists) shows that anti-fat prejudice parallels [18-22] and sometimes exceeds that reported in the general population [10]. For example, Schwartz et al. [20] found that even health professionals and obesity researchers attending an international conference on obesity displayed significant levels of implicit weight stigma. Similarly, O'Brien et al. [10] showed that pre-service physical education/exercise science students displayed greater levels of implicit weight stigma than a matched sample in other training disciplines, with weight stigma appearing to increase across training. Importantly, implicit measures of prejudice have been shown to be predictive of negative behaviors in both race bias [24] and weight bias research [25]. However, this relationship is controversial [26, 27].

Aside from the deleterious social consequences, anti-fat prejudice has negative physical and mental health implications for those who are targeted for such ill-treatment [22, 23, 28-34]. For example, Neumark-Stzainer et al. [28] found that weight-related teasing predicted future overweight status, disordered eating and binge eating 5 years later. Similarly, Eisenberg et al. [29, 30] showed that weight stigma/ teasing was associated with lower body image, higher depression and higher suicide ideation in adolescents. Other research shows that children subjected to anti-fat prejudice in physical activity settings reported less liking of sport and physical activity, and reported lower participation [31]. Clearly it is critical that effective approaches for the reduction of anti-fat prejudice be found and implemented in settings most in need. The present article reviews studies that have sought in the broadest sense to reduce anti-fat prejudice. Judgments of study effectiveness are made on the basis of significant changes in explicit and/or implicit anti-fat prejudice, rather than on changes in the psychological mechanisms thought underpin anti-fat prejudice (e.g. empathy, blame for weight status, belief that obesity is influenced by uncontrollable factors).

\section{Methodology}

A literature search for anti-fat prejudice reduction studies/interventions was conducted (completed April, 2009) using four online research databases (i.e. ISI Web of Knowledge, PsychInfo, PubMed, Scopus) and one internet search engine (Google). We also contacted individual researchers (via email and phone) who had published research in the area of anti-fat prejudice. Online searches included combinations of the terms 'weight', 'obesity', 'fat', 'anti-fat', 'size', 'bias', 'stigma', 'prejudice', 'stereotype', 'discrimination', 'bullying', 'hostility', 'intervention', 'program', 'reduction', 'prevention', 'acceptance', 'decreasing', 'modify', and 'reducing'. Reference lists of related publications were also examined for further sources not identified in online searches. This search strategy yielded 16 published works (some with multiple experiments) that could broadly be defined as anti-fat prejudice reduction studies/interventions [8, 35-49]. Because of the dearth of research in this area we have not applied strict exclusion/inclusion criteria but instead have sought to detail all of the work identified. The review does not include two dissertations [50, 51] nor a recent unpublished intervention [52]. The respective authors did not wish to have the data made public prior to formal publication.

Table 1 provides an overview of published studies. In addition to the shortage of research on this topic, there was a lack of consistency in the outcome measures used and in the theoretical and methodological approaches employed. For example, some studies [38, 47] measured and sought to reduce implicit anti-fat prejudice along with explicit anti-fat prejudice. Other research examined beliefs and fears about overweight and obesity as personal conditions alongside stereotypes of obese individuals [45]. Additionally, four of the studies used pre- and/or post-measure designs with no control or comparison group $[42,43,45,46]$ rather than more rigorous experimental [e.g. 44] or quasi-experimental (matched control group) research designs that allow inferences of causation to be made.

Clusters of studies that adopted common conceptual approaches were identified. A majority of studies sought to reduce anti-fat prejudice through the modification of knowledge and beliefs about the causes and controllability of overweight and obesity, either alone [8,35-37, 47] or in combination with other approaches [39, 41, 44, 45, 48]. Similarly, several studies sought to evoke empathy, acceptance and positive affect for those perceived as fat, either alone [42, 46, 47] or combined with other approaches [38, 39, 41, 43, 45, 48]. 


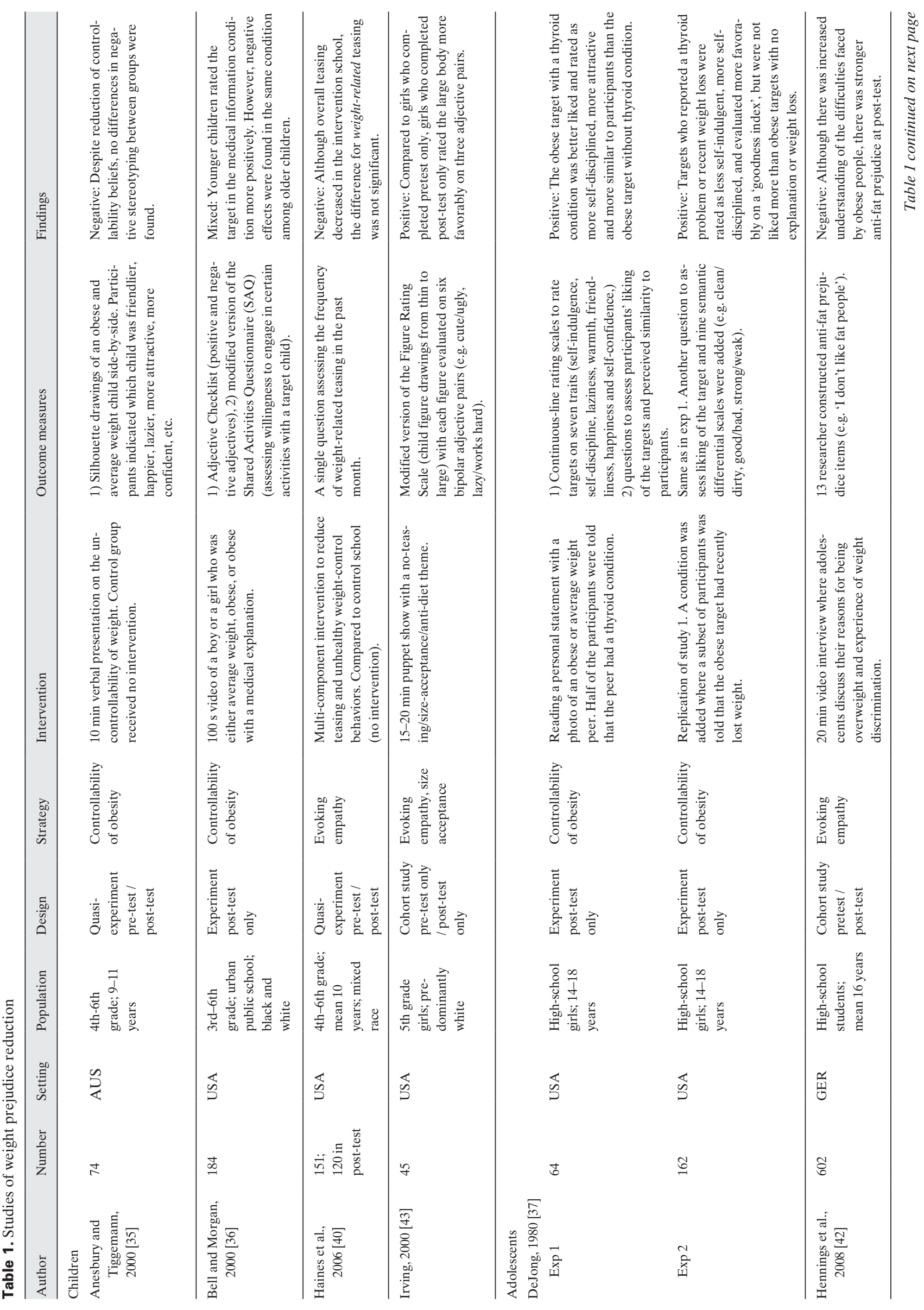




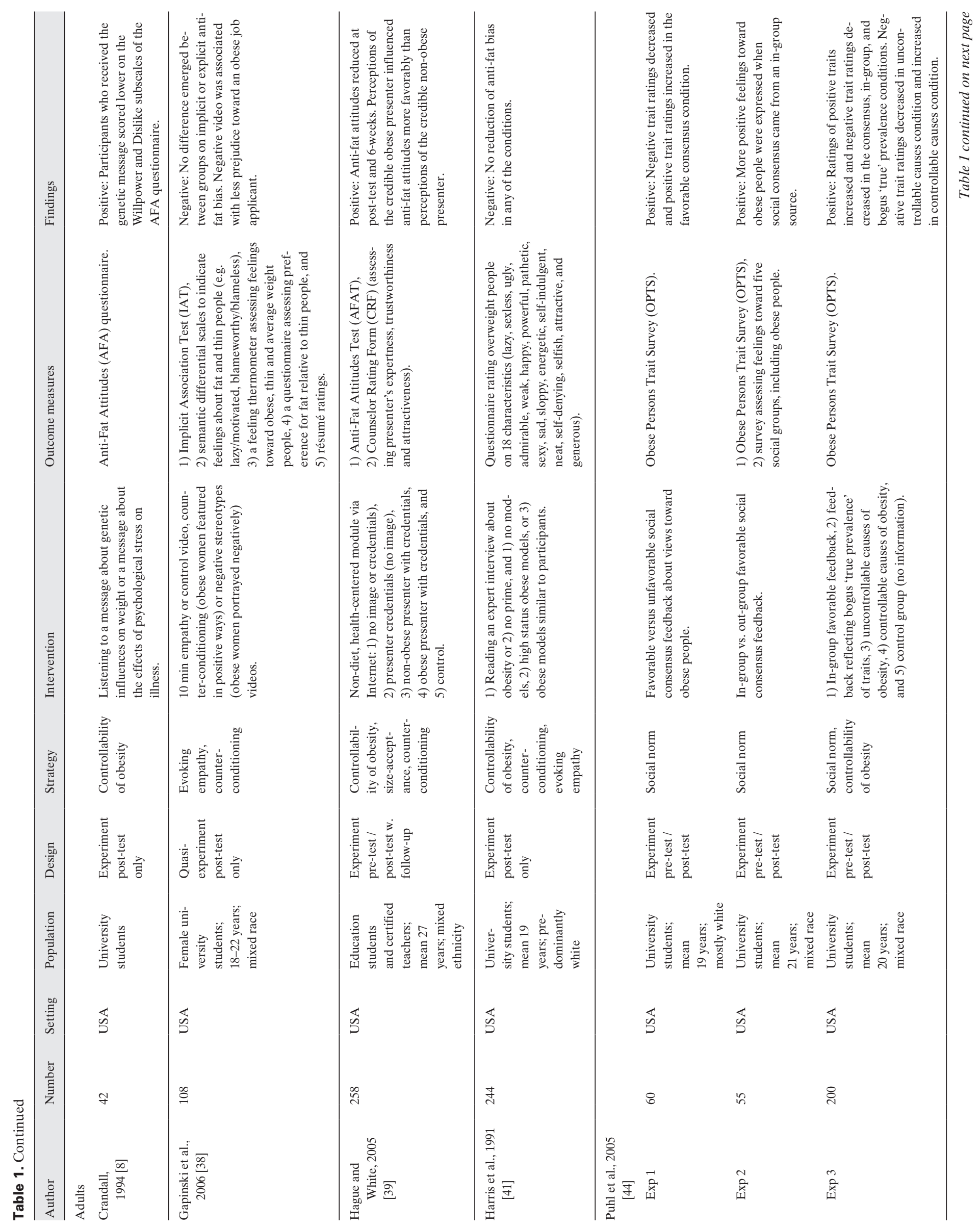




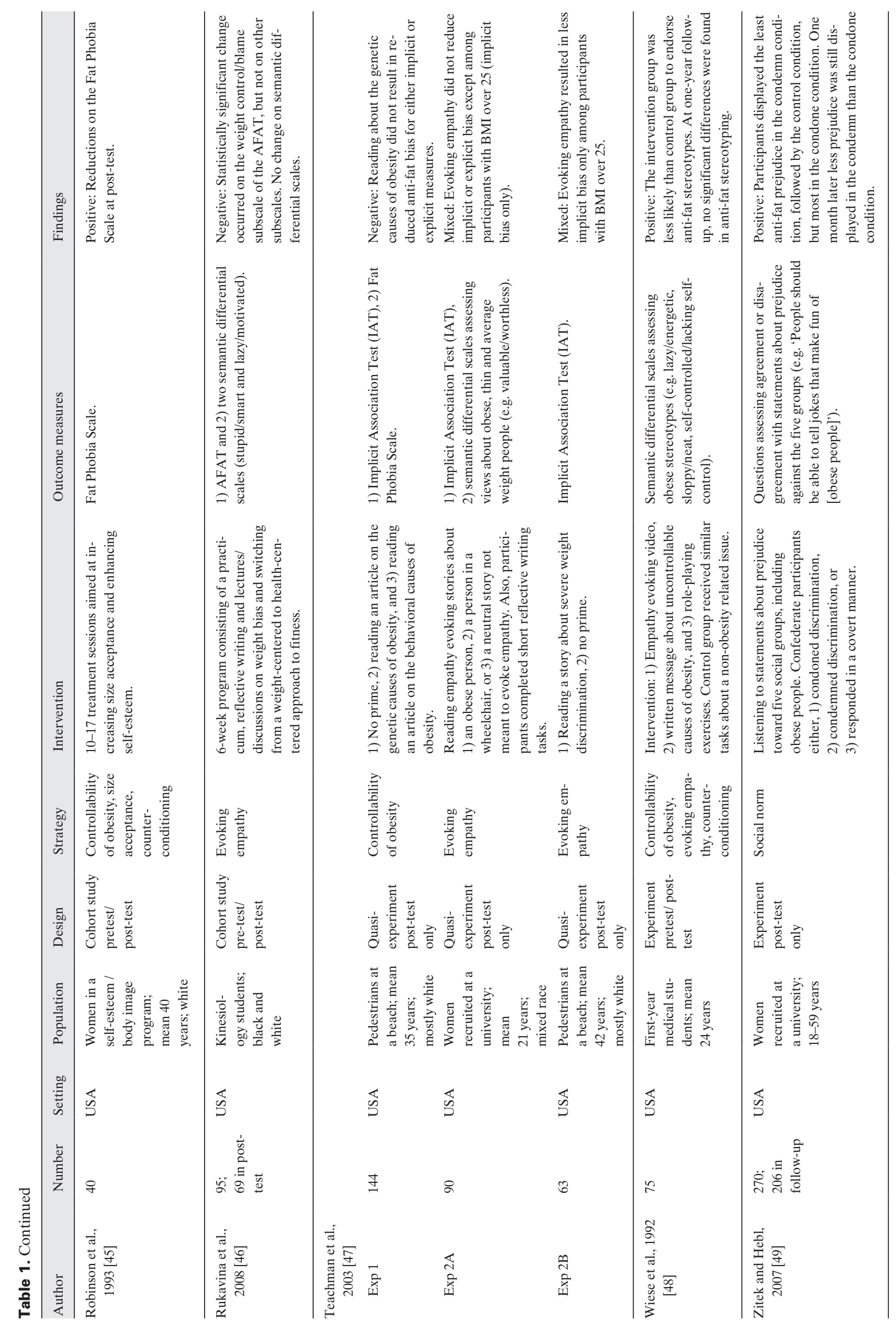


Examples of this approach are studies that presented obese people in a positive, or counter-stereotypical light, such as energetic and attractive (i.e. counter-conditioning) [38, 39, $41,45,48]$, or those that aimed to increase respect for weight diversity (i.e. size acceptance) [39, 43, 45]. Others explored the role of social influence in creating attitude change (e.g. social consensus and social norms) [44, 49]. Some studies used a combination of approaches for reducing prejudice [39, 40, 45, 48]. For example, Wiese et al. [48] sought to reduce anti-fat prejudice by manipulating causal attributions for obesity, evoking empathy and presenting an obese target in a counter-stereotypical light (e.g. as smart and attractive). Accordingly, we organize our descriptions of studies into the general themes of i) modifying beliefs about the causes and controllability of obesity, ii) promoting empathy, acceptance and liking, iii) manipulating social consensus and norms and iv) combined or multiple approaches. However, a more detailed description of the strategies used in each respective study can be found in table 1 . Following a review of the studies we offer thoughts regarding the quality of existing literature and future directions for interventions aimed at reducing anti-fat prejudice.

\section{Manipulating Beliefs about the Causes and Controllability of Obesity}

The research literature on anti-fat prejudice suggests that western society dislikes 'fat' people because they are perceived to contravene certain moral, ideological values (e.g. self-discipline, self-determination and the protestant work ethic). Despite evidence suggesting that weight status is determined by a complex interaction between biological and environmental factors, public opinion holds that overweight and obesity are controllable through dieting and physical activity. In line with the attribution value theory of anti-fat prejudice, anyone perceived to be 'fat' is thought to be lazy, self-indulgent, personally responsible for his or her condition, and worthy of ridicule $[8,53]$. Accordingly, investigators have sought to reduce anti-fat sentiment by changing beliefs about the causes and controllability of obesity.

DeJong [37] examined whether modifying beliefs about the causes and controllability of obesity could ameliorate negative judgments of obese adolescents. In experiment 1 , adolescent females were randomized to receive a descriptive personal profile and photo of female targets portrayed as obese or normal weight. Half of the profiles provided a medical explanation (a thyroid disorder) for their weight (obese target) or pale skin (normal weight target). DeJong [37] found that participants receiving profiles containing the thyroid explanation made more favorable judgments (i.e. less self-indulgent and more self-disciplined) and expressed greater liking of the obese target than for the obese target with no thyroid explanation. In a second experiment, DeJong added a third condi- tion where profiles described obese adolescents with and without a thyroid condition as having lost $25 \mathrm{lbs}$ in the past month. Despite differences in the perceived traits of obese targets (e.g. less self-indulgent, more self-disciplined), there was no significant difference in liking for obese targets providing medical explanations for their weight or for those who reported recent weight loss [37].

Two further studies $[35,36]$ manipulating perceived controllability of obesity were unsuccessful in reducing anti-fat prejudice in children. Bell and Morgan [36] randomly assigned children to one of six conditions where children were presented with a short (100 s) video of boys and girls portrayed as average weight, obese with no explanation for their weight, or obese with a medical explanation for their weight. Although the obese target with a medical explanation was rated as less blameworthy compared to the obese target with no explanation, only younger children (mean $=9$ years) showed positive changes in anti-fat attitudes and stereotypes. Importantly, providing a medical explanation did not increase the desire to actively engage with obese children. Indeed, older children expressed less desire to engage in academic activities with the obese target following the medical explanation.

Using a pre- and post-measure experimental design, Anesbury and Tiggemann [35] presented school children with genetic and biological reasons for differences in people's appearance (e.g. eye, skin, and hair color, height, body size and shape). As part of a class lesson, pictures of children at different body sizes (i.e. lean, average, and large body builds) were shown to participants. Children in the intervention group were provided with genetic and metabolic explanations for body size and shape differences and simultaneously told that diet and exercise alone do not determine one's weight. Despite significant changes in beliefs about the controllability of obesity, there was no significant reduction in negative stereotyping of obese children.

Studies in adult populations have yielded mixed success when manipulating attributions regarding the causes and controllability of obesity [8, 47]. Crandall [8] used set-point theory to provide information on the genetic and metabolic reasons for weight status and weight control to college students. Compared to controls, the intervention group reported lower anti-fat attitude scores. Specifically, the intervention group reported less explicit dislike of obese people and a reduced belief that obese people lack willpower. In another study, Teachman et al. [47; experiment 1] found that implicit and explicit anti-fat attitudes could be manipulated, however, not necessarily in the desired direction. When presenting evidence that obesity was determined by overeating and lack of exercise, participants displayed increased anti-fat prejudice (both implicit and explicit). However, genetic explanations for obesity did not produce less implicit or explicit anti-fat prejudice relative to controls [47]. 


\section{Comments on Modifying Beliefs about the Causes and Controllability of Obesity}

Studies manipulating attributions and beliefs about the causes and controllability of obesity provide less than encouraging evidence regarding their ability to reduce anti-fat prejudice. Three studies $[8,36,37]$ provided limited evidence that stereotypes can be influenced to the good. However, these studies did not use a pre- and post-experimental design; thus we cannot exclude the possibility that the results are due to socially desirable responding. Although Crandall's [8] results are encouraging because beliefs about the causes of obesity were not assessed at baseline differences in anti-fat attitudes between the intervention and control group cannot be directly attributed to changes in beliefs about obesity. Indeed, a more rigorous study design did not produce similar reductions in prejudice [35]. Additionally, even when modest differences in anti-fat prejudice were found, normal weight targets were still rated more positively on most characteristics than obese targets. It is worth suggesting for the studies conducted among children $[35,36]$ that although children may be willing to accept that obese peers are not responsible for their weight, they still may see them as being less fun and desirable than other peers. This lack of desire to engage with overweight peers [36] may be particularly salient in physical activity settings, where play often involves competition and having a larger peer on one's side may be perceived as a disadvantage. However, this would not explain the lack of desire to engage in academic pursuits. It may be that attributions of personal responsibility are not the primary reasons for children's bias against obese peers. It is possible that simple attractiveness mechanisms underpin lower positivity to obese peers compared to normal weight ones. Alternatively, medical explanations may elicit a desire to avoid infection or illness through contact with obese targets. Some have suggested that dislike of obesity as a condition may be underpinned by an evolutionary drive to avoid infection (pathogen avoidance; [54]).

The findings of Teachman et al. [47; experiment 1] of increased implicit prejudice following information on controllable causes of obesity, such as overeating and lack of physical activity, support the notion that attributions underpin anti-fat prejudice. They may also support to Crandall's and Eshleman's [55] Justification-Suppression Model (JSM) of prejudice, which amongst other things suggests that prejudice is more likely to be expressed (or not suppressed) when sufficient justification is provided for it. Suggesting that laziness and gluttony are the primary causes of obesity in Teachman et al. [47] may have provided the justification for the bias reported. The results for participants in the genetics condition, on the other hand, showed they were clearly not convinced by the material. This may be due to a lack of understanding of genetics or to the overexposure to material in the media and wider society, suggesting that diet and physical activity are the primary causes and cures for obesity.

\section{Evoking Empathy, Acceptance and Liking}

Increasing empathy and perspective taking has been shown to reduce prejudice in commonly stigmatized groups such as AIDS sufferers and African-Americans [56, 57]. Researchers have sought to apply this strategy to anti-fat prejudice via appeals to the more compassionate, social and accepting side of human nature [38, 41-43, 46, 47]. Teachman et al. [47; experiment $2 \mathrm{~A}$ ] randomized participants to one of three conditions (two stigmatized and one non-stigmatized control condition) where they read 3- to 4-page first-person narratives of social rejection and ridicule experienced by either obese women or wheelchair-bound women (stigmatized conditions), or a similar first-person account that was neutral in nature (control condition). Participants also completed a short reflective writing task following these empathy-evoking accounts. Manipulation checks showed that the obese and wheelchair conditions evoked significantly greater empathy than the control condition. However, post-intervention measures failed to show significant differences in either implicit or explicit antifat bias relative to the control group, except among overweight participants. In a second experiment, Teachman et al. [47; experiment 2B] found that evoking empathy was again generally ineffective; however, overweight participants in the obesity empathy-evoking condition did show slightly reduced fat bias relative to controls.

Two studies [38, 42] used short empathy-evoking video presentations in seeking to modify anti-fat prejudice. Hennings et al. [42] found that despite reporting greater understanding of the difficulties of being overweight, participants reported increased anti-fat prejudice following exposure to video presentations of overweight adolescents describing personal difficulties and experiences of discrimination. Gapinski et al. [38] employed both empathy evoking and counter-conditioning to reduce prejudice. Participants in a prejudice reduction condition were first primed with video clips of overweight people providing firsthand accounts of the difficulties, discrimination and feelings (e.g. helplessness, fear) they experience in connection to their weight. A control condition viewed videos of wildlife and cars. The intervention and control groups were further randomized to videos where they were presented with either a TV show clip of overweight women portrayed in a positive (e.g. smart, competent, energetic) or negative (e.g. unattractive, unprofessional, sluggish) light. The investigators took measures of empathy and feelings towards targets as well as implicit and explicit anti-fat bias. Participants were also presented (under the guise of an additional unrelated study) with two job résumés, featuring a more competent and a less competent applicant, which had photos of either an obese or normal weight woman attached. The participants were asked to make ratings of the target's job-related qualities and whether or not they would like the applicant as a roommate. On the whole, the intervention did not produce significant differences in implicit or explicit anti- 
fat bias. Interestingly, participants who saw the negative video showed less bias toward one of the obese applicants than participants who saw the positive video.

A study among kinesiology students [46] sought to raise consciousness regarding the difficulties faced by those who are overweight via lecture classes and contact with children during a fitness testing program. The course was unable to reduce anti-fat prejudice but did reduce negative beliefs about personal responsibility for obesity. However, the study did not have a control condition, and participants were not blinded to the study purpose; therefore, the study may have suffered from selection bias. In addition, the dropout rate in this study was high (27\%), and appropriate analyses (i.e. intention-to-treat analyses) were not conducted to account for attrition.

Other work using variants of the empathy-evoking and acceptance theme also yield mixed findings [41, 43]. Harris et al. [41] used an experimental design (post-measurements only) where intervention group participants initially read an interview given by an expert which contained factual information about obesity (prime). Participants were further assigned to conditions where they read interviews given by either highstatus overweight individuals, likable overweight individuals who were similar to the participants, or read no further interviews (control). No between-group differences in anti-fat prejudice were found. Finally, Irving [43] used a puppet program to promote size acceptance in young school children. The program delivered puppet shows and stories that promoted size acceptance messages and told children that teasing of others was wrong. Puppets constructed to look 'fat' were always portrayed as kind, caring and in a generally positive light. The program reported reductions in negative stereotyping of obese children, however, significant flaws in the measurement procedures of the study require that the results be viewed with caution. For example, of the 152 children participating only 45 (all girls and all in the oldest age bracket) had measures of anti-fat prejudice taken. Of these 45 girls, 20 had the measures taken only at baseline and were compared statistically to a different sample of 25 girls who only had postintervention measures of anti-fat prejudice taken. Thus, it cannot be determined whether true changes occurred.

\section{Comments on Evoking Empathy, Acceptance and Liking}

The weight of the evidence found here, and particularly from those studies that used stronger research designs [38, 41, 47], suggests that increasing empathy, acceptance and liking may not be an effective strategy for reducing anti-fat prejudice. This view needs to be tempered by the acknowledgement that there have been very few studies examining these strategies and fewer studies using rigorous and interpretable research methods. Gapinski et al. [38] note that a key assumption be- hind the principle of evoking empathy is the notion that the individual engages in perspective taking. It may be one thing to see an obese person's negative plight, but quite another to feel that plight personally and envisage oneself in it. Teachman et al.'s [47] findings perhaps hint at this perspective taking factor as they found slightly reduced anti-fat bias for overweight participants, but not for normal weight participants.

It is also possible that evoking empathy is a relatively ineffective strategy for anti-fat prejudice reduction because it emphasizes the negative sides of being overweight. Because antifat prejudice is in part attributable to perceiving obese individuals as 'weak' (e.g. lazy, unhealthy, lacking self-control) and portraying them as pity worthy may merely reinforce the 'weakness' stereotype. In challenging anti-fat prejudice, it may be more effective to invoke feelings of acceptance, equality and respect for obese individuals, rather than empathy or pity. However, since none of the studies reviewed here applied such prejudice reduction strategies (e.g. counter-conditioning, size acceptance) in isolation, only in conjunction with other approaches, it is impossible to draw firm conclusions about their potential effectiveness.

\section{Social Consensus and Social Norms}

The influence of social factors in changing people's views and beliefs is well understood [58], however, seldom used in the field of prejudice reduction [59]. Two studies have explored the role of social influence, specifically social consensus and social norms, in forming and reducing anti-fat prejudice [44, 49]. In three experiments, Puhl et al. [44] tested whether providing participants with phony feedback about the views of peers regarding the traits of and beliefs about obese people would influence the participants' subsequent views of obese people. All three studies took baseline measures of beliefs about obese people that were surreptitiously placed within a large battery of measures. One week later participants returned to complete measures again. In the first experiment, participants were randomized to receive feedback ostensibly about their peers' beliefs and trait ratings of obese people (consensus feedback). This feedback was framed to show that peers had either less or more negative beliefs about obese people, and assigned less or more negative/positive traits to obese people, than those reported by the participant. Providing consensus peer feedback of more positive attitudes reduced participants' assignment of negative traits to obese people and increased positive trait assignment. Beliefs about the personal controllability of obesity also decreased, despite no direct manipulation in that regard. Consensus feedback indicating more negative attitudes increased the assignment of negative traits to obese people. In a second experiment the anti-fat prejudice reduction effect was enhanced when receiving the feedback from ostensibly in-group members. In a third experiment Puhl et al. [44] compared five different prejudice 
reduction conditions; control (no information), positive consensus feedback from in-group members, false feedback on the true prevalence of obese people's positive and negative traits, a vignette describing the uncontrollable causes of obesity or a vignette describing controllable causes of obesity. Positive in-group consensus feedback and bogus feedback on obese trait prevalence both significantly reduced scores on the measures taken. It should be noted that the outcome measure used by Puhl et al. [44] was a measure of traits (positive and negative) that participants judged to be more or less descriptive of obese people, and not measures of dislike or antipathy toward obese people.

Zitek and Hebl [49] examined the role of social norm clarification in enhancing or reducing prejudice against several target groups, including obese people. Research assistants posed as either experimenters or confederates and invited college women from around campus to participate in the study. Participants were asked to verbally report their agreement with statements that either condemned or condoned discriminatory statements about a target group. However, the confederate was always chosen, ostensibly at random, to respond to the statements before the participant. The confederate responded in one of three predetermined ways, either verbally condemning or condoning the discriminatory statements or writing their response anonymously (control). Zitek and Hebl [49] found that participants were more likely to condemn or condone statements regarding discrimination (e.g. 'People should be able tell jokes that make fun of obese people') if they had first heard another peer condemn or condone the discriminatory statements. Importantly, the differences between conditions remained significant one month after the initial social norm manipulation.

\section{Comments on Social Consensus and Social Norms}

There have been very few studies examining the role of social consensus and social norm manipulations in anti-fat prejudice reduction. However, the evidence thus far suggests that this is a promising strategy for reducing anti-fat prejudice. The strength of social norms in directing and shaping attitudes and behavior has been shown in many domains other than prejudice research (e.g. drinking attitudes and behaviors [60]). Similarly, the influence of social norms and perceived consensus information on anti-fat attitudes fits with the model of Crandall and Eshleman (JSM) [55] in that underlying anti-fat prejudice may be suppressed when one perceives others' views as being less negative than one's own. However, this perspective suggests that underlying anti-fat prejudice is not necessarily being reduced, only the will and justification to express it. Regardless, the encouraging results from studies on social influence warrant further examination, perhaps alongside more naturalistic (e.g. class room based) prejudice reduction strategies. Importantly, Zitek and Hebl
[49] showed that social norm clarification may have enduring effects on the endorsement or condemnation of the expression of anti-fat prejudice.

\section{Combined or Multiple Strategies}

Four studies [39, 40, 45, 48] adopted combined or multi-strategy approaches, with two of these studies using the Elaboration Likelihood Model (ELM) [61] of attitude change as a framework for delivering interventions. Adopting the ELM, Wiese et al. [48] randomized 75 medical students to either control or experimental conditions to reduce anti-fat attitudes. Delivered in a 2-hour session, the experimental condition received information about the difficulties of weight loss from a credible source (video of an overweight, attractive nurse). Participants then reviewed research suggesting that obesity was largely the result of genetics, and, in a final phase intended to evoke empathy, students were asked to imagine being an obese woman and the difficulties associated with dating, making new friends or presenting to a new doctor for the first time. Changes in beliefs about the causes and difficulties associated with obesity and reduced endorsement of negative stereotypes (e.g. lazy, sloppy, lacking self-control) were reported; however, a close inspection of the data is required here. An examination of the participant numbers, cell means and standard deviations reported in table 2 [48, p. 863] suggests that after accounting for group differences at baseline the results may not reach significance (details of the statistical analysis conducted in this study are somewhat unclear).

Hague and White [39] also used the ELM in a 5-hour webbased intervention to reduce anti-fat prejudice in teachers and student teachers. A non-diet, health-centered educational program containing information on weight stigma and the etiology and treatment of obesity was presented to participants via online teaching software. The researcher also manipulated whether a presenter was present or not and whether the presenter was portrayed as overweight or not. Pre-test, post-test, and follow-up measures were taken using the Anti-Fat Attitudes Test (AFAT) [9]. All variants of the intervention produced reductions in AFAT scores that were still observed at a 6-week follow-up. The efficacy of this intervention does need to be considered within the context that the study had a $30 \%$ response rate and was advertised as an opportunity to learn about obesity and diversity in the classroom, and thus may have been comprised of a sample motivated to report reductions in anti-fat prejudice. Prejudiced people tend to avoid participating in studies on prejudice [62].

Robinson et al. [45] utilized multiple small group treatment sessions in an attempt to modify personal concerns regarding weight, body dissatisfaction, eating problems and the controllability of obesity. Group sessions focused on size acceptance with the hope of reducing personal distress and fat phobia. Although small pre-post-reductions in fat phobia 
were found, the study had limitations in the design that make interpretation difficult. For example, $87 \%$ of the sample were overweight or obese treatment-seeking women. Given the nature of the sample and aim of the intervention (to improve participants' own negative body-related cognitions and behaviors), it seems plausible that participant changes in fat phobia scores were more a reflection of positive self-affirmation rather than a reduction in prejudice towards others per se. Finally, although not primarily designed to reduce anti-fat prejudice, Haines et al. [40] examined changes in frequency of weight-related teasing following a school-based healthy eating and anti-teasing program. While this intensive multi-component program showed reductions in general teasing, weightrelated teasing (i.e., how often students were teased about their weight in the last month) was not significantly reduced.

\section{Comments on Combined or Multiple Strategies}

The evidence for the efficacy of combined or multiple strategies is encouraging but modest. It is difficult to determine whether the results reported for two of the studies $[39,48]$ were due to the adoption of a multi-strategy approach or the means of delivery. Specifically, two of the mixed strategy studies used the ELM [61] framework to facilitate attitude change, and thus the effects reported may have resulted from a structured and evidence-based attitude change methodology rather than from the adoption of combined strategies per se. Furthermore, since the study designs did not discriminate between the different bias reduction strategies, it is impossible to know which parts of the combination (e.g. size acceptance, counter-conditioning, changing attributions about obesity) were effective and which were not.

\section{General Conclusions and Suggestions for Future Research}

The purpose of this review was to identify published studies that have sought, broadly speaking, to reduce anti-fat prejudice. Most striking is the paucity of research efforts. This may reflect the relative infancy of the field, a lack of interest in addressing this type of prejudice or perhaps a tacit acceptance of anti-fat prejudice within society. Regardless, the studies reviewed here provide limited evidence for effective approaches to reducing different forms of anti-fat prejudice. What is clear is that a majority of the interventions were able to produce changes in the beliefs, attitudes and attributions that are thought to underpin and support anti-fat prejudice. For example, beliefs and knowledge about the uncontrollable causes of obesity were typically changed for the good; however, expression of anti-fat sentiment often remained. With other forms of prejudice (e.g. religious), negative attributions and stereotypes sometimes merely serve to provide a justification for the overt expression of underlying negative feelings toward the relevant target groups [55], but may not represent the true reason for the prejudice in the first place. This may also be the case for anti-fat prejudice. Similarly, increased empathy for obese individuals did not necessarily induce more liking of obese people. In addition, ideological views and personality traits (e.g. conservatism, authoritarianism, social dominance orientation), which have been shown to be related to anti-fat prejudice among other prejudices $[8,10]$, are rather stable and less likely to be amenable to change via the approaches reviewed here. The disjunct between changes in the proposed causes of anti-fat sentiment and changes in anti-fat prejudice itself is problematic.

Several reasons for the lack of success in changing anti-fat prejudice present themselves. First, it may be that factors other than those explored in the studies reviewed underpin the dislike of obese people. Recent research conducted by Park and colleagues [54] showed that fears about infection and disease were uniquely related to anti-fat prejudice, with an implicit association found between the concepts of obesity and disease. Kredl and colleagues [63] have also found that brain regions associated with disgust are activated when participants view images of stigmatized groups, including obese people. Other work premised on Social Identity Theory [64] suggests that anti-fat prejudice is linked to feelings about one's own appearance (body image). One potential way of feeling better about one's own appearance is to compare oneself to, and perhaps ridicule, someone perceived to be less attractive and desirable. In this regard anti-fat prejudice would be functional and rewarding for the protagonist $[65,66]$. If so, then changes in attributions regarding the causes of obesity are unlikely to reduce anti-fat prejudice. However, interventions that improve an individual's own body image and selfesteem may result in less derogation of obese individuals. Finally, if anti-fat prejudice is evoked through judgments based on unacceptable physical appearance and justified by attributions and stereotypes, then research examining the core emotion of disgust and its link with morality may be fruitful. Recent research on disgust suggests that those with greater propensity to be or feel disgusted display greater anti-gay sentiment $[67,68]$. A similar relationship may be found between disgust and anti-fat prejudice. How one might ameliorate this potential relationship is unclear. In summary, we suggest that researchers broaden the research base on the psychosocial reasons (conscious and unconscious) for anti-fat prejudice and seek to incorporate new findings into studies for reducing it.

Aside from the need for a broader examination of potential reasons for anti-fat prejudice, researchers need to adopt more rigorous research methods, particularly in terms of study design, population and setting. Our review found numerous problems with the designs of the studies reported here. Very few studies used randomized or experimental designs with pre-test and post-test measures. This limits the in- 
terpretability of any results that are reported, particularly for those studies which had no comparison groups. It is beyond the remit of this review to outline appropriate methodology for researchers; however, rigorous research designs are essential for the field to progress.

A related methodological problem lies with the measurement of anti-fat prejudice. It will be apparent to readers that there was little consistency in the choice of measures used to assess obesity stereotypes, general beliefs about obesity and anti-fat prejudice. Although reported as measures of prejudice, many of the measures do not assess dislike of fat people or antipathy, with a good many merely measuring stereotypes about obesity. This is problematic as one can hold unflattering stereotypes and beliefs about another group without disliking them. Indeed, unflattering stereotypes can be seen as endearing. A clear distinction therefore needs to be made between assessment of stereotypes and more meaningful measures of anti-fat antipathy and discrimination. This may require the development and psychometric testing of new measures that make clear distinctions between the assessment of general stereotypes and beliefs about fat people and actual antipathy toward them. Like other fields of prejudice research, more subtle measures of prejudice may need to be explored alongside existing measures, with real-world behavioral validation of such measures required. Implicit measures such as the implicit association test [20] appear to produce rather consistent findings in the anti-fat literature and were developed by social psychologists seeking more sensitive and potentially response bias-free measures of prejudice. While there is still some debate as to what implicit attitudes represent, and importantly, predict in terms of behavior (discrimination), we encourage researchers to employ these measures alongside explicit measures. Similarly, whilst laboratory testing with psychology students is often a pragmatic necessity, stealth anti-fat prejudice reduction studies do lend themselves well to structured classroom and university course settings. This may also aid in the assessment of anti-fat behavior through controlled observations and participant reports of weight-related teasing or exclusion. Only one study reviewed here examined anti-fat behavior, with rates of weight-based teasing remaining unchanged following an intensive school-based intervention [40]. Additionally, few studies established whether changes in anti-fat prejudice measures held outside of the laboratory or for any significant period of time. Similar problems have been identified in a recent review of nearly 1,000 interventions aimed at reducing prejudice toward various target groups (e.g. gays, blacks, religious groups [59]), with similar calls for redress made.

The evidence for the deleterious effects of anti-fat prejudice is fast accumulating with research on the prevalence and strength of anti-fat sentiment providing sobering reading for any concerned with social equality [17]. Unfortunately, research effort seeking to address this prejudice is lagging. Accordingly, it is critical that new approaches to anti-fat prejudice reduction be identified. We repeat calls [59] for more field-based prejudice reduction studies as these types of efforts allow the real-world efficacy and generalizability of such interventions to be assessed. Studies also need to adopt more rigorous research designs and methodologies. It would be tempting given the paucity of research in this area to welcome more studies regardless of design; however, the research reviewed here shows that the design of many of the studies makes the results uninterpretable.

\section{Disclosure}

The authors declared no conflicts of interest

\section{References}

1 International Obesity Task Force (ed): International Obesity Task Force Prevalence Data. $w w w$. iotf.org/database/index.asp. Accessed April 5, 2009.

2 Andreyeva T, Puhl RM, Brownell, KD: Changes in perceived weight discrimination among Americans, 1995-1996 through 2004-2006. Obesity 2008;16: $1129-1134$.

3 Latner JD, Stunkard AJ: Getting worse: the stigmatization of obese children. Obes Res 2003;11: 452-456.

4 Latner J, O'Brien KS, Durso LE, Brinkman LA, MacDonald T: Weighing obesity stigma: relative strength of different targets of bias. Int J Obes 2008; 32:1145-1152.

5. Puhl RM, Andreyeva T, Brownell KD: Perceptions of weight discrimination: Prevalence and comparison to race and gender discrimination in America. Int J Obes 2008;32:992-1000.

6 Neumark-Sztainer D, Story M, Harris T: Beliefs and attitudes about obesity among teachers and school health care providers working with adolescents. J Nutr Educ 1999;31:3-9.
7 Crandall CS: Do parents discriminate against their heavyweight daughters? Pers Soc Psychol Bull 1995; 21:724-735.

$\checkmark$ Crandall CS: Prejudice against fat people: ideology and self-interest. J Pers Soc Psychol 1994;66:882894.

9 Lewis RJ, Cash TF, Jacobi L, Bubb-Lewis C: Prejudice toward fat people: the development and validation of the Anti-Fat Attitudes Test. Obes Res 1997; 5:297-307.

10 O'Brien KS, Hunter JA, Banks M: Implicit antifat bias in physical educators: physical attributes, ideology, and socialisation. Int J Obes 2007;31:308314.

11 Roehling MV: Weight-based discrimination in employment: psychological and legal aspects. Pers Psychol 1999;52:969-1017.

12 Rudolph CW, Wells CL, Weller MD, Baltes BB: A meta-analysis of empirical studies of weight-based bias in the workplace. J Vocat Behav 2009;74:1-10.
3 Bagley CR, Conklin DN, Isherwood RT, Pechiulis DR, Watson LA: Attitudes of nurses toward obesity and obese patients. Percept Mot Skills 1989;68: 954.

14 Rand CW, MacGregor AM: Morbidly obese patients' perceptions of social discrimination before and after surgery for obesity. South Med J 1990;83: 1390-1395.

15 Hilbert A, Ried J: Obesity in print: an analysis of daily newspapers. Obes Facts 2009;2:46-51.

16 Greenberg BS, Eastin M, Hofschire L, Lachlan K, Brownell KD: Portrayals of overweight and obese individuals in commercial television. Am J Public Health 2003;93:1342-1348.

17 Puhl RM, Heuer CA: The stigma of obesity: a review and update. Obesity 2009;17:941-64.

18 Berryman DE, Dubale GM, Manchester DS, Mittelstaedt R: Dietetics students possess negative attitudes toward obesity similar to nondietetics students. J Am Diet Assoc 2006;106:1678-1682. 
19 Puhl RM, Wharton CM, Heuer C: Weight bias among dietetic students: implications for treatmen practices. J Am Diet Assoc 2009;109:438-444.

-20 Schwartz MB, Chambliss HO, Brownell KD, Blair $\mathrm{SN}$, Billington C: Weight bias among health professionals specializing in obesity. Obes Res 2003;11: 1033-1039.

21 Brown I: Nurses' attitudes towards adult patients who are obese: literature review. J Adv Nurs 2006; 53:221-232.

22 Davis-Coelho K, Waltz J, Davis-Coelho B: Awareness and prevention of bias against fat clients in psychotherapy. Prof Psychol Res Pract 2000;31: 682-684.

23 Maroney D, Golub S: Nurses' attitudes toward obese persons and certain ethnic groups. Percept Mot Skills 1992;75:387-391.

-24 Dovidio JK, Kawakami K, Gaertner SL: Implici and explicit prejudice and interracial interactions. J Pers Soc Psychol 2002;82:62-68.

25 Bessenoff GR, Sherman JW: Automatic and controlled components of prejudice toward fat people: evaluation versus stereotype activation. Soc Cogn 2000;18:329-353.

26 Blanton H, Jaccard J, Klick J, Mellers B, Mitchell G, Tetlock PE: Strong claims and weak evidence: reassessing the predictive validity of the IAT. J Appl Psychol 2009;94:567-582.

-27 O'Brien KS, Latner JD, Halberstadt J, Hunter JA, Anderson J, Caputi P: Do antifat attitudes predict antifat behaviors? Obesity 2008;16(suppl 2):S87S92.

28 Neumark-Sztainer DR, Wall MM, Haines JI, Story MT, Sherwood NE, van den Berg PA: Shared risk and protective factors for overweight and disordered eating in adolescents. Am J Prev Med 2007; 33:359-369.

29 Eisenberg ME, Neumark-Sztainer D, Story M: Associations of weight-based teasing and emotional well-being among adolescents. Arch Pediatr Adolesc Med 2003;157:733-738.

30 Eisenberg ME, Neumark-Sztainer DR, Haines JI, Wall MM: Weight-teasing and emotional wellbeing in adolescents: longitudinal findings from Project EAT. J Adolesc Health 2006;38:675-683.

-31 Faith MS, Leone MA, Ayers TS, Heo M, Pietrobelli A: Weight criticism during physical activity, coping skills, and reported physical activity in children. Pediatrics 2002;110:e23-e31.

32 Hebl MR, Xu J, Mason MF: Weighing the care: Patients' perceptions of physician care as a function of gender and weight. Int J Obes Relat Metab Disord 2003;27:269-275.

33 Hebl M, Xu J: Weighing the care: physicians' reactions to the size of a patient. Int J Obes Relat Metab Disord 2001;25:1246-1252.

34 Puhl RM, Latner JD: Stigma, obesity, and the health of nation's children. Psychol Bull 2007;133 557-580.

35 Anesbury T, Tiggemann M: An attempt to reduce negative stereotyping of obesity in children by changing controllability beliefs. Health Educ Res 2000;15:145-152.
36 Bell SK, Morgan SB: Children's attitudes and behavioral intentions toward a peer presented as obese: does a medical explanation for the obesity make a difference? J Pediatr Psychol 2000;25:137145.

37 DeJong W: The stigma of obesity: The consequences of naïve assumptions concerning the causes of physical deviance. J Health Soc Behav 1980;21:7587.

38 Gapinski KD, Schwartz MB, Brownell KD: Can television change anti-fat attitudes and behavior? J Appl Biobehav Res 2006;11:1-28.

39 Hague AL, White AA: Web-based intervention for changing attitudes of obesity among current and future teachers. J Nutr Educ Behav 2005;37:58-66.

40 Haines J, Neumark-Sztainer D, Perry CL, Hannan PJ, Levine MP: VIK (Very Important Kids): a school-based program designed to reduce teasing and unhealthy weight-control behaviours. Health Educ Res 2006;21:884-895.

41 Harris MB: Altering attitudes and knowledge about obesity. J Soc Psychol 1991;131:881-884.

42 Hennings A, Hilbert A, Thomas J, Siegfried W, Rief W: Reduction of stigma against obese people: effects of an educational film. Psychother Psychosom Med Psychol 2007;57:359-363.

43 Irving L: Promoting size acceptance in elementary school children: the EDAP puppet program. Int J Eat Disord 2000;8:221-232.

44 Puhl R, Schwartz MB, Brownell KD: Impact of perceived consensus on stereotypes about obese people: a new approach for reducing bias. Health Psychol 2005;24:517-525.

45 Robinson BE, Bacon JG, O'Reilly J: Fat phobia: measuring, understanding and changing anti-fat attitudes. Int J Eat Disord 1993;14:467-480.

46 Rukavina PB, Li W, Rowell MB: A service learning based intervention to change attitudes toward obese individuals in kinesiology pre-professionals. Soc Psychol Educ 2008;11:95-112.

47 Teachman BA, Gapinski KD, Brownell KD, Rawlins M, Jeyaram S: Demonstrations of implicit antifat bias: the impact of providing causal information and evoking empathy. Health Psychol 2003;22:6878.

48 Wiese HJ, Wilson JF, Jones RA, Neises M: Obesity stigma reduction in medical students. Int J Obes 1992;16:859-868.

49 Zitek EM, Hebl MR: The role of social norm clarity in the influenced expression of prejudice over time. J Exp Soc Psychol 2007;43:867-876.

50 Ciao A: Reducing obesity stigma: The effectiveness of cognitive dissonance and social consensus interventions. Unpublished dissertation, University of Hawaii at Manoa, 2009.

51 Grosko TA: Obesity stigma reduction. Unpublished dissertation, University of Manitoba, 2007.

52 O'Brien KS, Puhl RM, Latner JD, Mir AS, Hunter JA: Reducing anti-fat prejudice in pre-service health students: a randomized trial. (submitted for publication)
3 Crandall CS, D'Anello S, Sakalli N, Lazarus E, Nejtardt GW, Feather NT: An attribution-value model of prejudice: anti-fat attitudes in six nations. Pers Soc Psychol Bull 2001;27:30-37.

54 Park JH, Schaller M, Crandall CS: Pathogen-avoidance mechanisms and the stigmatization of obese people. Evol Hum Behav 2007:28:410-414.

55 Crandall CS, Eshleman A: A justification-suppression model of the expression and experience of prejudice. Psychol Bull 2003;129:414-446

56 Batson CD, Polycarpou MP, Harmon-Jones E, Imhoff HJ, Mitchener EC, Bednar LL, Klein TR, Highberger L: Empathy and attitudes: can feeling for a member of a stigmatized group improve feelings toward the group? J Pers Soc Psychol 1997;72: 105-118.

57 Vescio TK, Sechrist GB, Paolucci MP: Perspective taking and prejudice reduction: the mediational role of empathy arousal and situational attributions. Eur J Soc Psychol 2003;33:455-472.

58 Asch SE: Effects of group pressure upon the modification and distortion of judgments; in Guetskow H (ed): Groups, Leadership and Men. Pittsburgh, Carnegie Press, 1951, pp 177-190.

59 Paluck EL, Green DP: Prejudice reduction: what works? A review and assessment of research and practice. Annu Rev Psychol 2009;60:339-367.

60 Perkins HW: Social norms and the prevention of alcohol misuse in collegiate contexts. J Stud Alcohol Suppl 2002;14:164-172.

61 Petty RE, Cacioppo JT: Communication and Persuasion: Central and Peripheral Routes to Attitude Change. New York, Springer, 1986.

62 Farley J: Majority-Minority Relations, 4th ed. Englewood Cliffs, Prentice Hall, 2000.

63 Krendl AC, Macrae CN, Kelley WM, Fugelsang JA, Heatherton TF: The good, the bad, and the ugly: an investigation of the functional anatomic correlates of stigma. Soc Neurosci 2006;1:5-15.

64 Tajfel H, Turner JC: The social identity theory of inter-group behavior; in Worchel S, Austin LW (eds): Psychology of Intergroup Relations. Chicago, Nelson-Hall, 1986, pp 2-24.

65 O'Brien KS, Hunter JA, Halberstadt J, Anderson $\mathrm{J}$ : Body image and explicit and implicit anti-fat attitudes: the mediating role of physical appearance comparisons. Body Image 2007;4:249-256.

66 O’Brien KS, Caputi P, Minto R, Peoples G, Hooper C, Kell S, Sawley E: Upward and downward physical appearance-related comparisons: development of a measure and examination of predictive qualities. Body Image 2009;6:201-206.

67 Inbar Y, Pizarro DA, Bloom P: Conservatives are more easily disgusted than liberals. Cogn Emot 2009;23:714-725.

68 Inbar Y, Pizarro DA, Knobe J, Bloom P: Disgust sensitivity predicts intuitive disapproval of gays. Cogn Emot 2009;9:435-439. 